\title{
Left Common Carotid Artery Agenesis with Hypoplastic Left Internal Carotid Artery Originating from the Aortic Arch: A Rare Anomaly Presenting with Internal Carotid Artery Aneurysm and Subarachnoid Hemorrhage
}

\author{
Mandeep S. Ghuman ${ }^{1} \quad$ Vivek Gupta $^{1} \quad$ Shankhneel Singh ${ }^{1} \quad$ S. Dhandapani ${ }^{2} \quad$ N. Khandelwal ${ }^{1}$
}

\footnotetext{
1 Department of Radiodiagnosis and Neurosurgery, Postgraduate Institute of Medical Education and Research, Chandigarh, India

${ }^{2}$ Department of Neurosurgery, Postgraduate Institute of Medical Education and Research, Chandigarh, India
}

\begin{abstract}
Address for correspondence Vivek Gupta, DM, Department of Radiodiagnosis and Neurosurgery, Postgraduate Institute of Medical Education and Research, Sector 12, Chandigarh, India (e-mail: drvivekgupta.pgi@gmail.com).
\end{abstract}

J Clin Interv Radiol ISVIR 2017;1:49-52.
Abstract
Keywords
- CCA agenesis
- Hypoplastic ICA
- arch of aorta
- subarachnoid hemorrhage

\begin{abstract}
Most arch anomalies are asymptomatic and detected incidentally on imaging or on autopsy. Occasionally, such anomalies can manifest clinically when associated with another vascular pathology such as an intracranial aneurysm. In this report, we describe a rare case of agenesis of the left common carotid artery with separate origin of the left internal carotid artery and the external carotid artery from the arch discovered on digital subtraction angiography performed during the evaluation of subarachnoid hemorrhage. Knowledge of such anomalies and radiographic appearance is essential for interventional neuroradiologist in planning treatment of such cases.
\end{abstract}

\section{Introduction}

Anomalies of the origin of great vessels of the neck are well known and adequately described in the literature. These anomalies may be associated with other conditions and have bearing on treatment planning. We present one of the rare anomalies of the aortic arch in which the left internal carotid artery (ICA) originated from the aortic arch. A brief description of the case along with embryological basis is discussed.

\section{Case Report}

A 30-year-old woman presented with a history of sudden onset severe headache and loss of consciousness. A cranial computed tomography $(\mathrm{CT})$ scan revealed subarachnoid hemorrhage. Subsequent cerebral CT angiography revealed an aneurysm of the ICA at its supraclinoid segment. The intracranial ICA was narrowed in caliber and multiple calcifications were noted at the neck of the aneurysm (- Fig. 1A). There was no dissection or intraluminal/mural thrombus. In addition, the left bony carotid canal was small compared with the right one (-Fig. 1B). A cerebral angiography was performed to better characterize the aneurysm and the parent artery and to evaluate the crossflow across the circle of Willis. The right ICA was normal in its origin and caliber. There was good cross flow to the left through the anterior communicating artery (ACA) during a venous delay of only 0.5 seconds. Right vertebral angiogram showed a normal posterior circulation, with patent left posterior communication artery having slow flow (-Fig. 2A, B). However, there was difficulty during attempted cannulation of left common carotid artery (CCA). Next to the brachiocephalic artery, a branch from the arch was catheterized thinking it was the left CCA; however, angiography revealed it to be the left external carotid artery (ECA). This artery was unusually large in caliber, branching in the cervicofacial region at $\mathrm{C} 3$-vertebral level with no communication with the intracranial circulation ( $\mathbf{- F i g} . \mathbf{2 C}$ ). After the ECA, the next vessel arising from the arch was catheterized and angiography performed. This showed a small caliber artery, having a long, nonbranching but excessively loopy course in the neck, entering the cranial cavity through the petrous canal and 


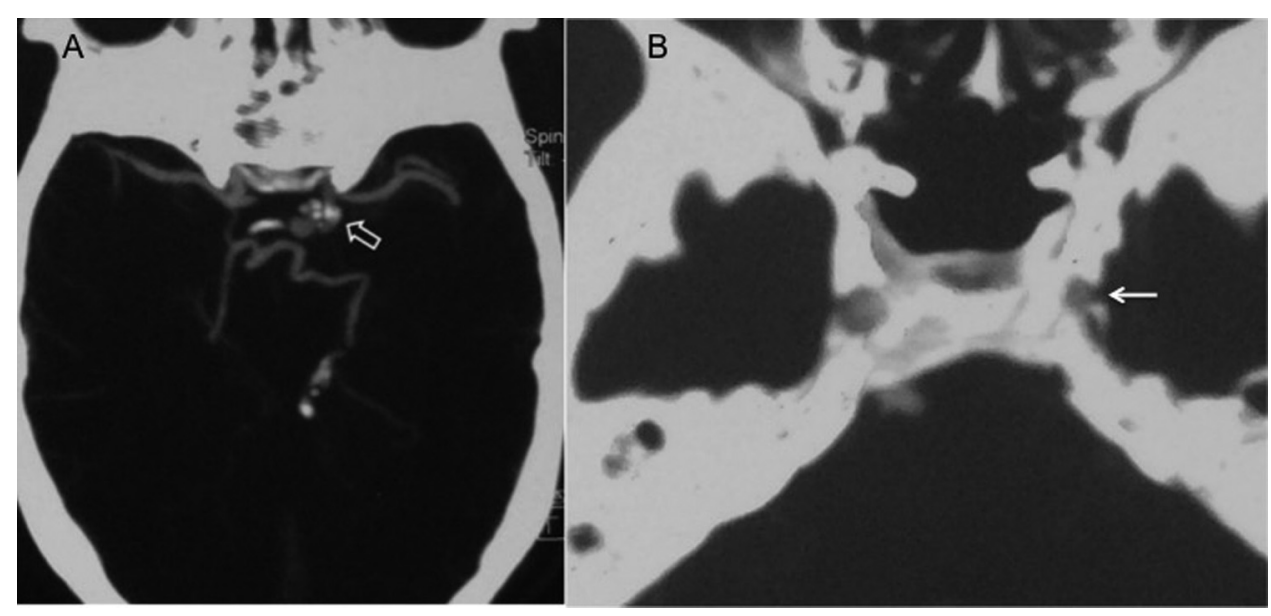

Fig. 1 (A, B) Axial maximum intensity projection image from computed tomography (CT) angiography data. (A) Image shows a posteriorly directed saccular aneurysm (arrow) arising from the supraclinoid segment of left internal carotid artery. Multiple calcifications are seen around the neck of the aneurysm. (B) Axial CT image at the level of the carotid canal shows a small left carotid canal.

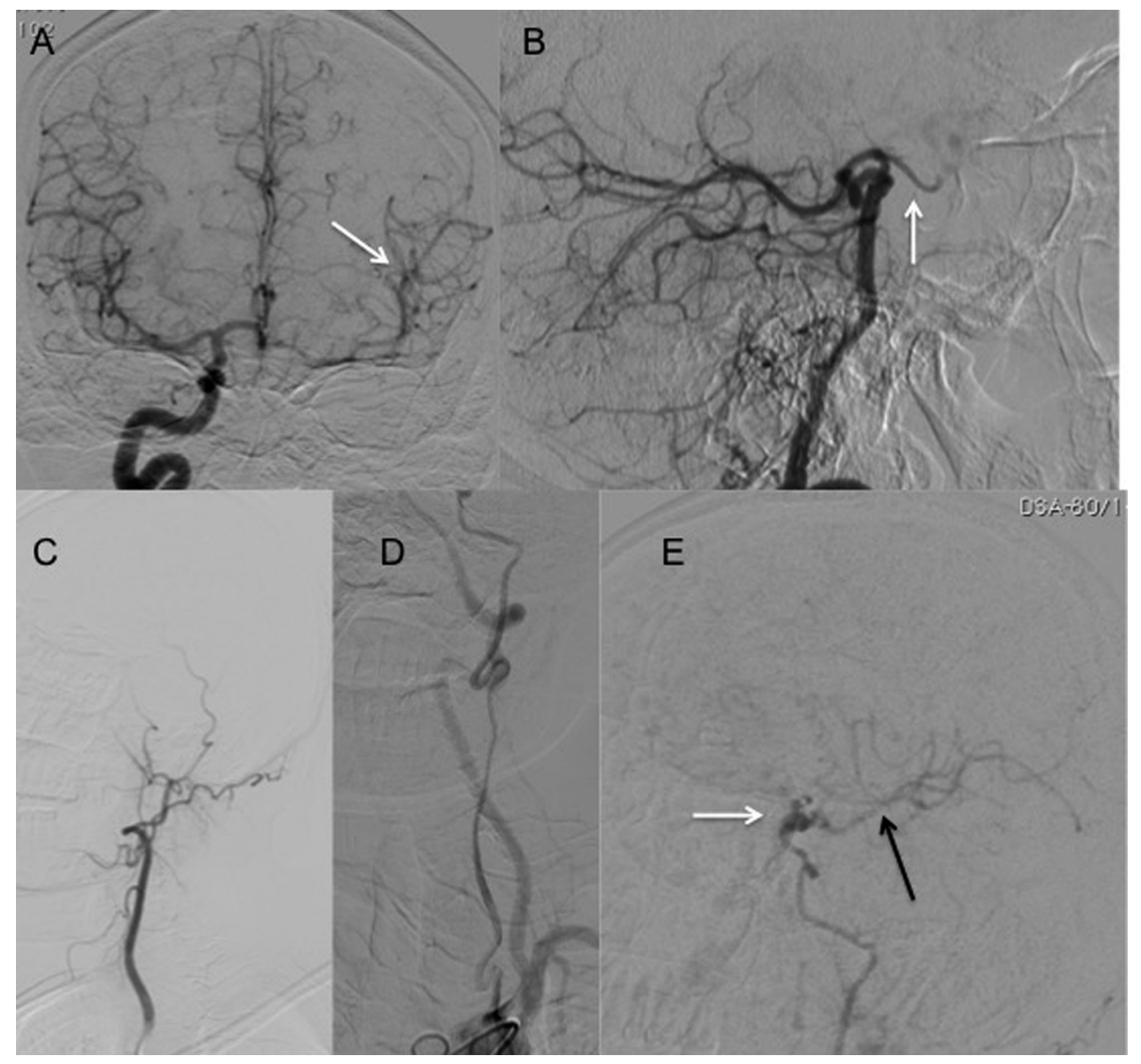

Fig. 2 (A-E) Right internal carotid angiography. (A) shows good collateral flow (arrow) to the left anterior circulation. (B) Right vertebral angiography shows a patent left posterior communication artery (arrow) with poor flow. (C) Angiography of the left external carotid artery (ECA) in an oblique view shows a prominent ECA with no intracranial communications. (D, E) The left internal carotid artery has a direct aortic origin with poor intracranial flow (arrow). 

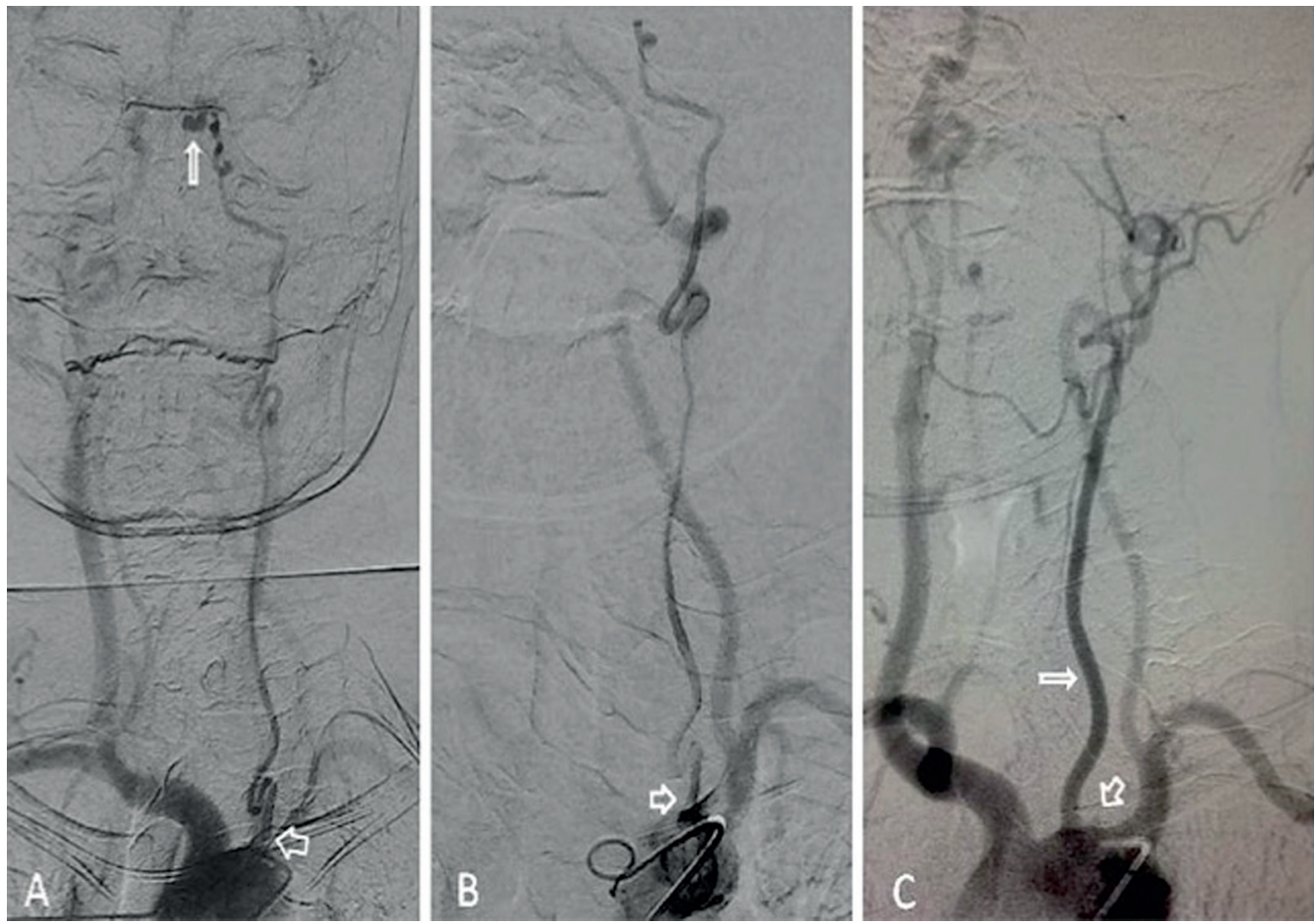

Fig. 3 (A-C) Digital subtraction angiography (DSA) images of the arch of aorta reveal small caliber left internal carotid artery (ICA) originating directly from the arch of the aorta (short arrow; A, B) along with a saccular aneurysm arising from its intracranial segment (long vertical arrow, A). (C) Follow-up DSA of the aortic arch 1 year after surgical clipping of the aneurysm demonstrates complete occlusion of the left ICA just beyond its origin (short arrow). Relatively lager caliber left ECA (thin long arrow, C) can be seen originating separately from the arch, proximal to the ICA origin.

conforming to the usual ICA course and distribution thereafter (-Fig. 2D). There was faint opacification of the left middle cerebral artery (-Fig. 2E). The left ACA was not visualized. A saccular aneurysm directed posteromedially and inferiorly was found arising from the supracliniod segment of this anomalous and small ICA ( - Fig. 3A, B). The aneurysm measured approximately $7 \times 5 \mathrm{~mm}$ and was arising from the medial wall of the left ICA. There was marked dextroscoliosis of the dorsal spine. In view of the small caliber, tortuous and loopy course of the cervical segment of this anomalous ICA harboring the aneurysm, endovascular therapy was not considered feasible and the patient underwent surgical clipping of the aneurysm. The postoperative course was uneventful and patient was discharged after 2 weeks in fully conscious and ambulatory state. Follow-up cerebral angiography performed 1 year after the surgery showed complete occlusion of left ICA just beyond its origin from the arch of the aorta ( $\mathbf{- F i g . ~ 3 C ) . ~ A d e q u a t e ~ c r o s s f l o w ~ w a s ~}$ documented through the anterior and posterior communicating arteries; however, there was no opacification of the clipped aneurysm. The patient was asymptomatic without any focal neurologic deficit. The patient reported one episode of headache and mild transient sensory symptoms on the right side of the body a few months after aneurysmal clipping for which no treatment was given.

\section{Discussion}

Agenesis of the CCA has been reported in fewer than 25 cases. ${ }^{1}$ However, congenital absence of left CCA is an ex- tremely rare anomaly in which the internal and external carotid arteries originate directly from the aortic arch. Only a few cases have been reported in the literature. ${ }^{2-4}$ Angiographic features and embryologic development hypothesis were described for the first time by Lie in $1968 .^{5}$

In embryonic development, the aortic arch and the great vessels develop in a sequential manner, secondary to the formation and regression of the paired vascular arches that connect the ventral aorta with the paired dorsal aorta. ${ }^{3}$ The third arches are precursors of the carotid system. The left fourth arch remains continuous with the aortic sac and the left dorsal aorta to form the left aortic arch in postnatal life. ${ }^{3,4}$ According to the widely accepted view, failure of regression and persistence of the ductus caroticus (portion of the embryonic dorsal aorta between the third and fourth arches) lead to the development of separate origin of the ICA. This happens in conjunction with involution of the third aortic arch that normally persists and becomes the CCA and proximal ICA. 1,2,4 An embryonic vessel, the ventral pharyngeal artery, which is a direct branch of the aortic sac, forms the ECA. The origin of the ECA migrates from the aortic sac for a variable distance along the third arch during descent of the heart. ${ }^{3,4}$ An alternate hypothesis postulates that a failure of the ECA to migrate laterally and join the ICA leads to the development of separate origins of internal and external carotid arteries from the arch of the aorta. ${ }^{1,4}$

When the CCA is absent on the right side, the ICA and ECA arise from the right subclavian artery, whereas when this anomaly occurs on the left side, the ICA and ECA arise from the aortic arch. $^{2}$ Various congenital anomalies have been 
associated with the agenesis of the CCA. These include cervical aortic arch, double aortic arch, right aortic arch, aberrant right subclavian artery, persistent trigeminal artery, and persistent proatlantal intersegmental artery. ${ }^{4}$

Normally, the ICA is larger in caliber than the ECA. The small caliber of the ICA in the index case could be assumed due to the persistence of a smaller ductus caroticus that formed the ICA. ${ }^{4}$ Agenesis of the CCA and the occurrence of an aneurysm has been described in a single case where a saccular aneurysm was found involving the extracranial segment of the ICA, which originated directly from the arch of the aorta. ${ }^{6}$

In conclusion, an intracranial saccular aneurysm arising from an atretic ICA associated with agenesis of the CCA has not been reported previously. This case highlights the complex variation in the embryological development of the cervicocranial vasculature. Detailed diagnostic assessment of the arch and cervical vessels in addition to the intracranial vascular imaging in selected cases can reveal unexpected anatomical or morphological variations and significant implications on therapeutic decisions (surgery or endovascular management).

\section{Funding}

There is no financial contribution from any source.

\section{Conflict of Interest}

There is no conflict of the interest and this report has not been published or sent for review to any other journal.

\section{References}

1 Yim SJ, Ryu JH, Baik JS, Kim JY, Park JH, Han SW. Common carotid artery agenesis: duplex ultrasonographic findings. J Clin Neurol 2009;5(3):149-150

2 Maybody M, Uszynski M, Morton E, Vitek JJ. Absence of the common carotid artery: a rare vascular anomaly. AJNR Am J Neuroradiol 2003;24(4):711-713

3 Horowitz M, Bansal S, Dastur K. Aortic arch origin of the left external carotid artery and type II proatlantal fetal anastomosis. AJNR Am J Neuroradiol 2003;24(3):323-325

4 Cakirer S, Karaarslan E, Kayabali M, Rozanes I. Separate origins of the left internal and external carotid arteries from the aortic arch: MR angiographic findings. AJNR Am J Neuroradiol 2002;23(9): 1600-1602

5 Lie TA. Congenital Anomalies of the Carotid Arteries: Including the Carotid-Basilar and Carotid-Vertebral Anastomoses. An Angiographic Study and a Review of the Literature. Amsterdam, the Netherlands: Excerpta Medica; 1968:30-35

6 Kocogullar CU, Becit N, Erkut B, Kocak H. A case report of an abnormal configuration of the branches of aortic arch with an internal carotid artery aneurysm. Eur J Vasc Endovasc Surg 2005;9(3):44-45 